\title{
Simulation of Throughput in UMTS Networks with Different Spreading Factors
}

\author{
Robert Akl \\ Department of Computer Science and Engineering \\ University of North Texas \\ Denton, Texas 76207 Email: rakl@cse.unt.edu
}

\author{
Anurag Arepally \\ Department of Computer Science and Engineering \\ University of North Texas \\ Denton, Texas 76207 Email: aa0118@cse.unt.edu
}

\begin{abstract}
We design and implement a local session ${ }^{1}$ admission control (SAC) algorithm for third-generation wireless networks which allows for the simulation of network throughput for different spreading factors and various mobility scenarios. The design of the SAC algorithm uses global information; it incorporates the session arrival rates and the user mobilities across the network and guarantees the users' quality of service as well as prespecified blocking probabilities. On the other hand, its implementation in each cell uses local information; it only requires the number of sessions currently active in that cell. A global SAC algorithm is also implemented and used as a benchmark since it is inherently optimized and uses global information in making every session admission decision; it yields the best possible performance but has an intensive computational complexity. Using simulation, we determine the network throughput, and show that our optimized local SAC algorithm achieves almost the same performance as our global SAC algorithm at a fraction of the computational cost for pre-specified blocking probabilities and quality of service requirements and spreading factor values of $256,64,16$, and 4 .
\end{abstract}

\section{INTRODUCTION}

Wideband Code Division Multiple Access (WCDMA) is an enhancement to the Code Division Multiple Access (CDMA) technology with an increase in the voice capacity and high speed data transfer (up to $2 \mathrm{Mbps}$ ) for Third Generation (3G) Cellular Systems. Universal Mobile Telecommunications System (UMTS) defines WCDMA as the preferred access technique for multimedia traffic.

The heterogeneous nature of sessions combined with intracell and intercell interferences drastically affects the capacity of a UMTS network, which leads to designing a Session Admission Control (SAC) algorithm to maintain the quality of service $(\mathrm{QoS})$ and maximum resource utilization [1]. Generally, SAC algorithms fall under two approaches - local and global [2], [3]. The session admittance for a local SAC algorithm considers only a single cell while its design may look at the whole network. A global SAC algorithm takes the entire network into account to make every session processing decision. Although this is an optimal solution, the amount of computational complexity and scalability of global SAC algorithms pose serious disadvantages.

Our contributions are two-fold. First, we design, analyze, and simulate a local SAC algorithm for UMTS networks by

\footnotetext{
${ }^{1}$ The term "session" is used instead of "call" since when sending data in packets one may not really have a call.
}

formulating an optimization problem that maximizes the network throughput for different spreading factors using signalto-interference constraints as lower bounds. The solution to this problem is the maximum number of sessions that can be admitted in each cell. The design is optimized for the entire network, and the implementation is simple and considers only a single cell for admitting a session. Second, we also design, analyze, and simulate a global SAC algorithm to be used as a benchmark for various services. Our global SAC algorithm, even though it is inherently optimized for any session arrival rate profile, bears the overhead of real time interference calculations for every arriving session and has a computational complexity that is exponential in the number of cells. On the other hand, our local SAC algorithm performs its optimization calculations in advance and uses the solution obtained for session admission control decisions. Consequently, our local SAC algorithm is much simpler to implement. The simulation results show that the optimized local SAC achieves almost the same performance as our global SAC in terms of network throughput for spreading factor values of $256,64,16$, and 4 .

The remainder of this paper is organized as follows. In section II, we present our mobility model. In sections III and IV, we describe our global and local SAC algorithms, respectively. In section $\mathrm{V}$, spreading factors are introduced for UMTS networks. In section VI, simulation results are presented. Finally, the conclusions drawn from this paper are summarized in section VII.

\section{Mobility Model}

There are several mobility models that have been discussed in the literature [4]-[6]. These models have ranged from general dwell times for calls to ones that have hyper-exponential and sub-exponential distributions. For the SAC problem that we are investigating here, however, such assumptions makes the problem mathematically intractable. The mobility model that we use is presented in [7] where a session stops occupying a cell either because user mobility has forced the session to be handed off to another cell, or because the session is completed.

The session arrival process to cell $i$ with service $g$ is assumed to be a Poisson process with rate $\lambda_{i, g}$ independent of other session arrival processes. The session dwell time is a random variable with exponential distribution having mean $1 / \mu$, and it is independent of earlier arrival times, session 
durations and elapsed times of other sessions. At the end of a dwell time a session may stay in the same cell, attempt a handoff to an adjacent cell, or leave the network. We define $q_{i i, g}$ as the probability that a session in progress in cell $i$ (with service $g$ ) remains in cell $i$ after completing its dwell time. In this case a new dwell time that is independent of the previous dwell time begins immediately. Let $q_{i j, g}$ be the probability that a session in progress in cell $i$ after completing its dwell time moves to cell $j$. If cells $i$ and $j$ are not adjacent, then we make $q_{i j, g}=0$. We denote by $q_{i, g}$ the probability that a session in progress in cell $i$ leaves the network.

\section{Global SAC Algorithm}

We start by presenting the global SAC algorithm for multicell UMTS networks and give details of its implementation using a simulator model.

\section{A. Feasible Session Configuration}

Consider a multi-cell UMTS network with spread signal bandwidth of $W$, information rate of $R_{g}$ bits/s, received signal $S_{g}$ (at the serving base station assuming perfect power control), activity factor of $v_{g}$, and background noise spectral density of $N_{0}$. Assuming a total of $G$ services and $M$ cells, the bit energy to interference density ratio in cell $i$ with service $g$ is given by [8]

$$
\begin{aligned}
\left(\frac{E_{b}}{I_{0}}\right)_{i, g}= & \frac{\frac{S_{g}}{R_{g}}}{N_{0}+\frac{S_{g}}{W}\left[\sum_{g=1}^{G} n_{i, g} v_{g}+\sum_{j=1, j \neq i}^{M} \sum_{g=1}^{G} n_{j, g} v_{g} \kappa_{j i, g}-v_{g}\right]}, \\
& \text { for } i=1, \ldots, M, \quad g=1, \ldots, G,
\end{aligned}
$$

where $n_{i, g}$ is the number of sessions in cell $i$ (with service $g$ ) and $\kappa_{j i, g}$ is the per-session relative inter-cell interference factor from cell $j$ to cell $i$. To achieve a required bit error rate, we must have $\left(\frac{E_{b}}{I_{0}}\right)_{i, g} \geq \tau_{g}$ for some constant $\tau_{g}$. Thus, the number of simultaneous sessions in each cell must satisfy

$$
\sum_{g=1}^{G} n_{i, g} v_{g}+\sum_{j=1, j \neq i}^{M} \sum_{g=1}^{G} n_{j, g} v_{g} \kappa_{j i, g}-v_{g} \leq c_{e f f}^{(g)},
$$

where

$$
c_{e f f}^{(g)}=\frac{W}{R_{g}}\left[\frac{1}{\tau_{g}}-\frac{R_{g}}{S_{g} / N_{0}}\right] .
$$

A set of sessions $\mathbf{n}$ satisfying the above equations is said to be a feasible session configuration, i.e., one that satisfies the $\left(\frac{E_{b}}{I_{0}}\right)$ constraints. In this work, we assume perfect power control and refer the reader to [9] for an extension to imperfect power control.

\section{B. Simulator Model}

The total simultaneous sessions that guarantee the quality of service for session admission requirements must satisfy

$$
\begin{array}{r}
C_{i, g}(t)=n_{i, g}(t)+I_{i, g}(t) \leq c_{e f f}^{(g)}, \\
\quad \text { for } \quad i=1, \ldots, M, \quad g=1, \ldots, G .
\end{array}
$$

for every time $t$ where $I_{i, g}$ is the total relative inter-cell interference at cell $i$ caused by every user in the network with service $g$. A new session or a handoff session arriving in cell $i$ (with service $g$ ) is blocked if this session leads to a violation of any of the above inequalities, i.e., causing interference that no longer meets the $\left(\frac{E_{b}}{I_{0}}\right)$ constraints from (2).

1) Session Arrival and Admission Module: The module is comprised of two parts, session arrival control and session admission control. The session arrival control generates sessions for the UMTS network whereas the session admission control processes these sessions and tries to admit them depending on the total offered traffic for a particular cell while maintaining the QoS for the whole network. The total offered traffic $\rho_{i, g}(t)$ to cell $i$ with service $g$ for time $t$ is calculated as

$$
\begin{aligned}
& \rho_{i, g}(t)=\lambda_{i, g}(t)+\nu_{i, g}(t), \\
& \text { for } i=1, \ldots, M, \quad g=1, \ldots, G,
\end{aligned}
$$

where $\nu_{i, g}(t)$ are the sessions that moved to cell $i$ with service $g$ at time $t-1$. Note that $\nu_{i, g}(1)=0$, for $i=1, \ldots, M$ and $g=1, \ldots, G$. The session arrival control then selects each session randomly for a randomly chosen cell, and passes it to the session admission control along with the location of this new session.

In order to determine if the session can be admitted, the session admission control computes $C_{i, g}$ to check if the conditions given by (4) will still hold if the session is allowed to enter the network. Traditionally, the total interference contributed by a cell has been viewed as an approximation, determined by simply multiplying the number of sessions in that cell by the average per-session inter-cell interference factor offered by that cell [10]. In other words, a session placed anywhere within a cell generates the same amount of inter-cell interference. However, we also present a second more realistic approach and calculate the actual interference as a function of the actual distance of each user from each base station. We use this approach in our simulations.

2) Average Interference: If average interference is used (and a uniform user distribution is assumed), let $F_{j i, g}$ be the average per-session inter-cell interference factor with service $g$ from cell $j$ to cell $i$ [11]. $F_{j i, g}$ are elements in a three dimensional matrix $F$ with $i, j=1, \ldots, M$ and $g=1, \ldots, G$. Consequently, the total relative average inter-cell interference experienced by cell $i$ is simply the summation of the product of number of sessions $n_{j, g}$ in cell $j$ with service $g$ and their respective per-session interference factor $F_{j i, g}$. Since matrix $F$, which has a computational complexity of $O\left(M^{2} G\right)$, can be computed in advance, the above calculation is adequately fast since it requires only $M$ lookups in the matrix. However, the interference caused by a user is independent of its location within a given cell. The computational complexity for the implementation of the global SAC algorithm using average interference is $O(M G)$.

3) Actual Interference: If the actual distance of each user $k$ from its serving base station is used to calculate interference, the matrix $F$ cannot be used. Instead, a new matrix $U$ is computed and updated in order to account for the actual 
increase in interference due to admitting a new session:

$$
\begin{gathered}
U[j, i, g](t)=U[j, i, g](t-1)+\left(U_{j i, g}\right)_{k}, \\
\text { for } i j=1, \ldots, M, \quad g=1, \ldots, G,
\end{gathered}
$$

where, $U[j, i, g](t)$ contains the current total relative actual inter-cell interference exerted by cell $j$ to cell $i$ and $\left(U_{j i, g}\right)_{k}$ is the relative actual interference offered by user $k$ in cell $j$ to cell $i$ with service $g$. Now, the new set of $C_{i, g}(t)$ is calculated as

$$
\begin{gathered}
C_{i, g}(t)=n_{i, g}(t)+\sum_{j=1, j \neq i}^{M} U[j, i, g](t), \\
\text { for } \quad i=1, \ldots, M, \quad g=1, \ldots, G .
\end{gathered}
$$

The computational complexity for the implementation of the global SAC algorithm using actual interference is $O\left(M^{2} G\right)$.

If the inequalities in (4) still hold for the new $C_{i, g}$ 's, then the session is allowed to enter the network, otherwise the session is rejected. We would like to point out that since our simulator does not incorporate an intra-cell mobility model, the simulator places users randomly in a cell. At the end of dwell time, if there is no handoff then the session is placed again randomly in the cell and it's new interference is updated which may result in the session being dropped.

\section{Optimized Local SAC Algorithm}

\section{A. Admissible Session Configuration}

Recall that in the global SAC algorithm, a session arriving in cell $i$ with service $g$ is accepted if and only if the new state is a feasible state. Clearly that SAC algorithm requires global state, i.e., the number of sessions in progress in all the cells of the network. In order to simplify the SAC algorithm, we consider only those SAC algorithms which utilize local state, i.e., the number of sessions in progress in the current cell. To this end we define a state $\mathbf{n}$ to be admissible if

$$
n_{i, g} \leq N_{i, g} \text { for } i=1, \ldots, M \text { and } g=1, \ldots, G,
$$

where $N_{i, g}$ is a parameter which denotes the maximum number of sessions with service $g$ allowed to be admitted in cell $i$. Clearly the set of admissible states is a subset of the set of feasible states. The blocking probability for cell $i$ with service $g$ is then given by

$$
B_{i, g}=B\left(T_{i, g}, N_{i, g}\right)=\frac{T_{i, g}^{N_{i, g}} / N_{i, g} !}{\sum_{k=0}^{N_{i, g}} T_{i, g}^{k} / k !},
$$

where $T_{i}=\rho_{i, g} / \mu_{i, g}=\rho_{i, g} / \mu\left(1-q_{i i, g}\right)$ is the Erlang traffic in cell $i$ with service $g$. We note that the complexity to calculate the blocking probabilities in (9) is $O(M G)$.

Once the maximum number of sessions (for different services) that are allowed to be admitted in each cell, $\mathbf{N}$, is calculated (this is done offline and described in the next section), the local SAC algorithm for cell $i$ will simply compare the number of sessions with service $g$ currently active in cell $i$ to $N_{i, g}$ in order to accept or reject a new arriving session. Thus our local SAC algorithm is implemented with a computational complexity that is $O(1)$.

\section{B. Calculation of $\mathbf{N}$}

Our local SAC algorithm is constructed as follows. We formulate a constrained optimization problem in order to maximize the throughput subject to upper bounds on the blocking probabilities and a lower bound on the signal-tointerference constraints in (2). The goal is to optimize the utilization of network resources and provide consistent GoS while at the same time maintaining the QoS, $\beta_{g}$, for all the sessions for different services $g$. This approach for designing the SAC also allows different thresholds for blocking to be set in individual cells.

The throughput of cell $i$ consists of two components: the new sessions that are accepted in cell $i$ minus the forced termination due to handoff failure of the handoff sessions into cell $i$ for all services $g$. Hence the total throughput, $H$, of the network is

$$
H(\mathbf{B}, \rho, \lambda)=\sum_{i=1}^{M} \sum_{g=1}^{G}\left\{\lambda_{i, g}-B_{i, g} \rho_{i, g}\right\},
$$

where $\mathbf{B}$ is the vector of blocking probabilities and $\lambda$ is the matrix of session arrival rates.

In this optimization problem the arrival rates are given and the maximum number of sessions that can be admitted in all the cells are the independent variables. This is given in the following

$$
\max _{\mathbf{N}} H(\mathbf{B}, \rho, \lambda),
$$

subject to $B\left(T_{i, g}, N_{i, g}\right) \leq \beta_{g}$,

$$
\begin{aligned}
& \sum_{\substack{g=1 \\
\text { for }}}^{G} N_{i, g} v_{g}+\sum_{j=1, j \neq i}^{M} \sum_{g=1}^{G} N_{j, g} v_{g} \kappa_{j i, g}-v_{g} \leq c_{e f f}^{(g)}, \\
& \text {. }
\end{aligned}
$$

The optimization problem in (11) is solved offline to obtain the values of $\mathbf{N}$.

In a network's coverage area the session arrival rate profile, $\lambda$, will change from time to time. By solving the optimization problem in (11), one can compute the values of $\mathbf{N}$ for different session arrival rate profiles and store these in the mobile switching center along with the time periods of the corresponding profiles. A dynamic local SAC algorithm can then be implemented whereby the optimized values of $\mathbf{N}$ are used during each corresponding time period. This is reminiscent of the dynamic nonhierarchical routing which was introduced in the mid 1980's in the long distance AT\&T network [12].

\section{SPREAding FACTORS}

In UMTS networks, just like CDMA networks, communication from a single source is separated by channelization codes, i.e., the dedicated physical channel in the uplink and the downlink connections within one sector from one mobile station (MS) to a Base Station (BS). The Orthogonal Variable Spreading Factor (OVSF) codes, which were originally introduced in [13], were used to be channelization codes for UMTS. The use of OVSF codes allows the orthogonality and spreading 
TABLE I

UPLINK DPDCH DATA RATES.

\begin{tabular}{|c|c|c|}
\hline DPDCH Spreading Factor & Channel bit rate (Kbps) & Maximum session data rate \\
\hline 256 & 15 & $7.5 \mathrm{Kbps}$ \\
128 & 30 & $15 \mathrm{Kbps}$ \\
64 & 60 & $30 \mathrm{Kbps}$ \\
32 & 120 & $60 \mathrm{Kbps}$ \\
16 & 240 & $120 \mathrm{Kbps}$ \\
8 & 480 & $240 \mathrm{Kbps}$ \\
4 & 960 & $480 \mathrm{Kbps}$ \\
4, with 6 parallel codes & 5740 & $2.8 \mathrm{Mbps}$ \\
\hline
\end{tabular}

TABLE II

THE LOW MOBILITY PROBABILITIES.

\begin{tabular}{|c||c|c|c|}
\hline$\left\|\mathcal{A}_{i}\right\|$ & $q_{i j, g}$ & $q_{i i, g}$ & $q_{i, g}$ \\
\hline \hline 3 & 0.020 & 0.240 & 0.700 \\
\hline 4 & 0.015 & 0.240 & 0.700 \\
\hline 5 & 0.012 & 0.240 & 0.700 \\
\hline 6 & 0.010 & 0.240 & 0.700 \\
\hline \hline
\end{tabular}

factor (SF) to be changed between different spreading codes of different lengths.

Table I shows the approximation of the maximum session data rate with $\frac{1}{2}$ rate coding for different values of Dedicated Physical Data Channel. Our simulation results for network throughput are for spreading factor values of $256,64,16$, and 4.

\section{Simulation Results}

The following results have been obtained for a twenty-seven cell UMTS network. We assume the following for the analysis. The bit energy to interference ratio threshold is $7.5 \mathrm{~dB}$. The activity factor is 0.375 . The processing gain $\frac{W}{R_{g}}$ is $6.02 \mathrm{~dB}$, $12.04 \mathrm{~dB}, 18.06 \mathrm{~dB}, 24.08 \mathrm{~dB}$ for Spreading Factor (SF) of 4, 16,64 , and 256 , respectively.

Three mobility scenarios: no mobility, low mobility, and high mobility of users are considered. The following parameters are used for the no mobility case: $q_{i j, g}=0, q_{i i, g}=0.3$ and $q_{i, g}=0.7$ for all cells $i$ and $j$. Tables II and III show respectively the mobility characteristics and parameters for the low and high mobility cases. In all three mobility scenarios, the probability that a session leaves the network after completing its dwell time is 0.7 . Thus, regardless of where the session originates and mobility scenario used, the average dwell time of a session in the network is constant. In the numerical results below, for each SF value, we analyze the average throughput per cell by dividing the results from (11) by the total number of cells in the network and multiplying by the maximum data rate in Table I.

\section{A. UMTS Throughput Optimization with SF of 256}

First, we set SF to 256, which is used to carry data for the control channels. Fig. 1 shows the throughput per cell for a blocking probability from $1 \%$ to $10 \%$. The throughput for our local SAC is within $5 \%$ of the throughput for the global SAC algorithm for SF equal to 256.
TABLE III

THE HIGH MOBILITY PROBABILITIES.

\begin{tabular}{|c||c|c|c|}
\hline$\left\|\mathcal{A}_{i}\right\|$ & $q_{i j, g}$ & $q_{i i, g}$ & $q_{i, g}$ \\
\hline \hline 3 & 0.100 & 0.000 & 0.700 \\
\hline 4 & 0.075 & 0.000 & 0.700 \\
\hline 5 & 0.060 & 0.000 & 0.700 \\
\hline 6 & 0.050 & 0.000 & 0.700 \\
\hline
\end{tabular}

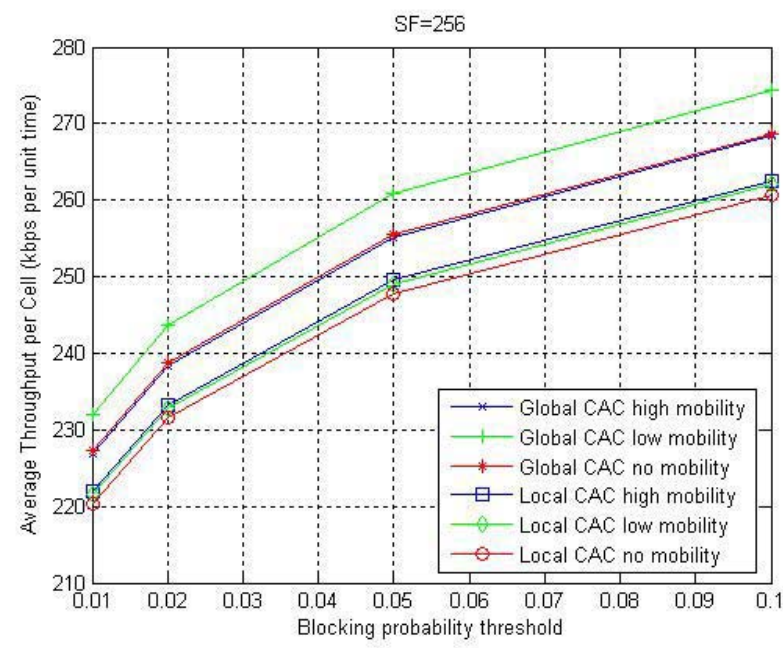

Fig. 1. Average throughput in each cell for $\mathrm{SF}=256$.

\section{B. UMTS Throughput Optimization with SF of 64}

Next, we set SF to 64, which is used for voice communication. As a result of lowering the SF to 64 , the number of possible concurrent connections within one cell is also decreased. Because the throughput is calculated based on the number of simultaneous connections between MSs and BSs, the lower trunking efficiency [14] leads to lower throughput as shown in Fig. 2. The throughput for our local SAC is within $4 \%$ of the throughput for the global SAC algorithm for SF equal to 64 .

\section{UMTS Throughput Optimization with SF of 16}

Next, we set SF equal to 16 , which is used for $64 \mathrm{Kbps}$ data communication. The resulting throughput, as shown in Fig. 3, is much lower compared to the case with SF equal to 64 or 256. The throughput for our local SAC is within $3 \%$ of the throughput for the global SAC algorithm for SF equal to 16.

\section{UMTS Throughput Optimization with SF of 4}

Next, we set SF equal to 4, which is used for $256 \mathrm{Kbps}$ data communication between BSs and MSs. The throughput for all three mobility cases are almost identical as shown in Fig. 4. The throughput for our local SAC is within $2 \%$ of the throughput for the global SAC algorithm for SF equal to 4 .

\section{CONCLUSIONS}

We design and implement a local SAC algorithm for UMTS networks and simulate network throughput for different spreading factors $(256,64,16$, and 4$)$ and various mobility 


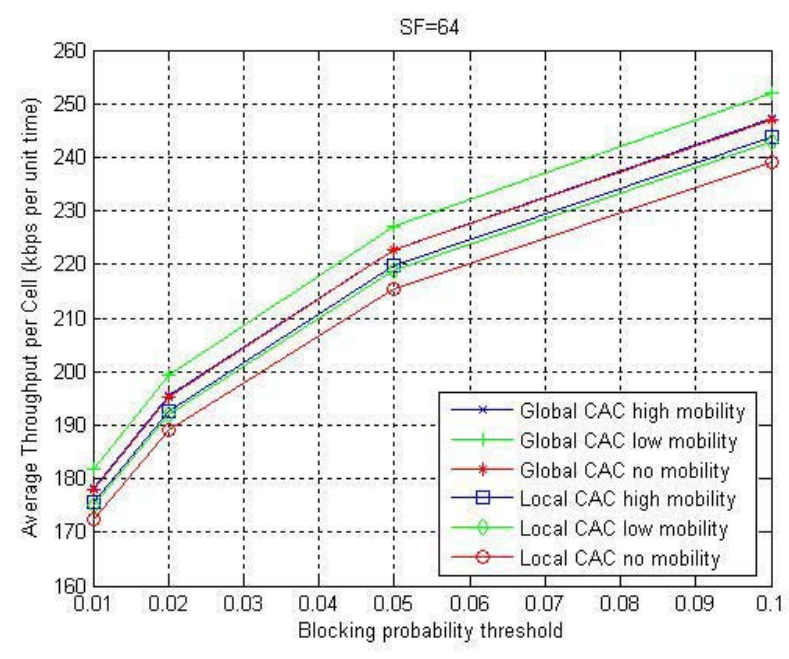

Fig. 2. Average throughput in each cell for $\mathrm{SF}=64$.

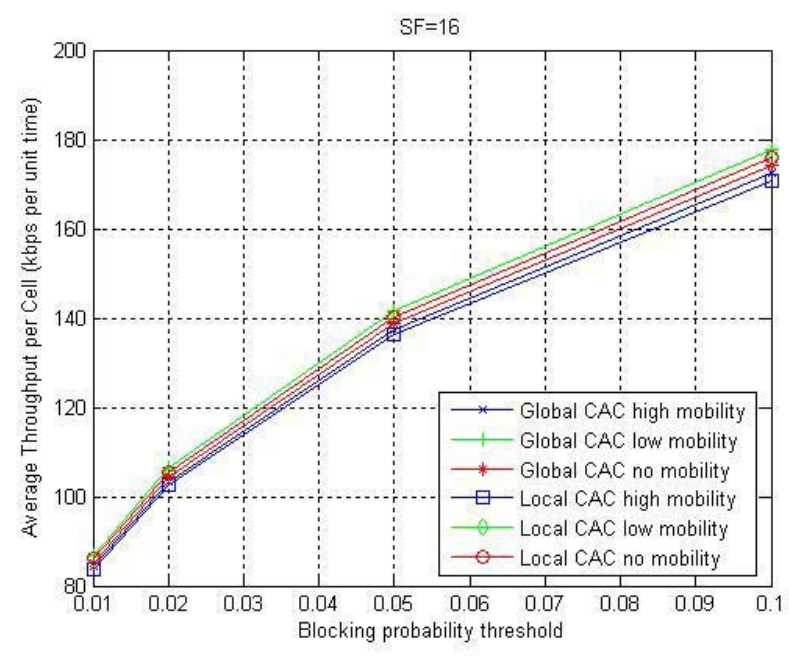

Fig. 3. Average throughput in each cell for $\mathrm{SF}=16$.

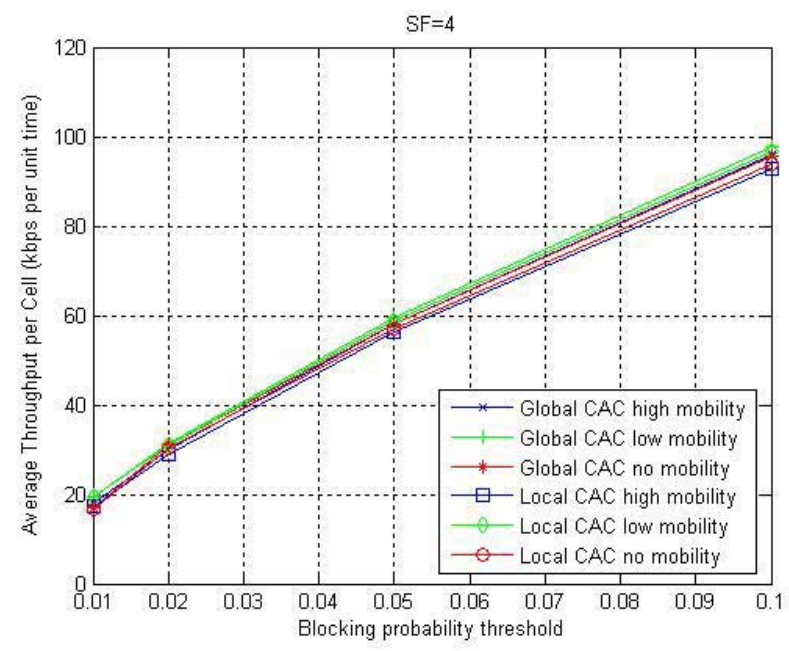

Fig. 4. Average throughput in each cell for $\mathrm{SF}=4$. scenarios (no, low and high mobility). The design of the local SAC algorithm uses global information; it incorporates the session arrival rates and the user mobilities across the network and guarantees the users' quality of service as well as pre-specified blocking probabilities. On the other hand, its implementation in each cell uses local information; it only requires the number of sessions currently active in that cell. A global SAC algorithm is also implemented and used as a benchmark since it is inherently optimized and uses global information in making every session admission decision; it yields the best possible performance but has an intensive computational complexity. The computational complexity of the implementation of the global SAC algorithm using average interference is $O(M G)$ and using actual interference is $O\left(M^{2} G\right)$ where $M$ is the total number of cells and $G$ is the total number of services, while the computational complexity of the implementation of our optimized local SAC is $O(1)$. Our global SAC algorithm is inherently optimized, and therefore it is expected to perform better than our local SAC algorithm. Our results show that the difference between our optimized local SAC algorithm's performance and our global SAC algorithm's performance is small enough (less than 5\%) to justify the small tradeoff in throughput for the huge reduction in computational complexity and feasibility for implementation in networks of large size.

\section{REFERENCES}

[1] J. Ye, X. Shen, and J. W. Mark, "Call admission control in wideband CDMA cellular networks by using fuzzy logic," IEEE Transactions on Mobile Computing, pp. 129-141, March/April 2005.

[2] T. Tugcu and C. Ersoy, "A novel call admission scheme based on interference for DS-CDMA systems," IEEE Veh. Technol. Conf., pp. 109-114, 2000.

[3] L. Zhu, Z. Ling, and S. Wu, "Call admission control in multiservices CDMA systems," IEEE Commun., Circuits and Systems and West Sino Expositions, vol. 1, pp. 177-181, July 2002.

[4] T. Tugcu and C. Ersoy, "Application of a realistic mobility model to call admissions in DS-CDMA cellular systems," IEEE Veh. Technol. Conf., vol. 2, pp. 1047-1051, Spring 2001.

[5] P. Orlik and S. Rappaport, "On the handoff arrival process in cellular communications," Wireless Networks, vol. 7, pp. 147-157, 2001.

[6] Y. Fang, I. Chlamtac, and Y.-B. Lin, "Modeling PCS networks under general call holding time and cell residence time distributions," IEEE/ACM Trans. on Networking, vol. 5, pp. 893-906, 1997.

[7] C. Vargas, M. Hegde, and M. Naraghi-Pour, "Implied costs for multirate wireless networks," J.Wireless Networks, vol. 10, pp. 323-337, May 2004.

[8] R. Akl, M. Hegde, and M. Naraghi-Pour, "Mobility-based CAC algorithm for arbitrary traffic distribution in CDMA cellular systems," IEEE Trans. Veh. Technol., vol. 54, pp. 639-651, March 2005.

[9] R. Akl and S. Nguyen, "Capacity allocation in multi-cell UMTS networks for different spreading factors with perfect and imperfect power control," IEEE Consumer Commun. and Networking Conf., January 2006.

[10] K. Gilhousen, I. Jacobs, R. Padovani, A. Viterbi, L. Weaver, and C. Wheatley, "On the capacity of a cellular CDMA system," IEEE Trans. Veh. Technol., vol. 40, no. 2, pp. 303-312, May 1991.

[11] A. Viterbi, CDMA Principles of Spread Spectrum Communication. Addison-Wesley, 1995.

[12] G. Ash, R. Cardwell, and R. Murray, "Design and optimization of networks with dynamic routing," Bell System Technical Journal, vol. 60, pp. 1787-1820, 1981.

[13] A. F., S. M., and O. K., "Tree-structured generation of orthogonal spreading codes with different lengths for forward link of DS-CDMA mobile radio," Electr. Lett., vol. 33, pp. 27-28, January 1997.

[14] T. Rappaport, Wireless Communications: Principles and Practice, $2 e$. Prentice Hall, 2002. 